\title{
Prognostic Value of C-Reactive Protein to Albumin Ratio in Patients Resuscitated from Out-of-Hospital Cardiac Arrest
}

\author{
Tuğba Bingol Tanriverdi ${ }^{1}$, Gulcin Patmano ${ }^{1}$, Firdevs Tugba Bozkurt ${ }^{1}$, Bedri Caner Kaya ${ }^{1}$, \\ and Mehmet Tercan ${ }^{1}$ \\ ${ }^{1}$ Sanliurfa Mehmet Akif Inan Training and Research Hospital
}

February 27, 2021

\begin{abstract}
Background: Despite major advances in basic and advanced life supports, patients who survived from out of hospital cardiac arrest (OHCA) has still poor prognosis. Several inflammatory parameters have been used to determine early and long-term prognosis in patients with OHCA. C-reactive protein-to-albumin ratio (CAR) is also a novel marker of systemic inflammation. To our knowledge, there is no study evaluating the clinical importance of CAR in OHCA patients. Aims: To evaluate the effect of CAR on mortality in patients with OHCA. Methods: A total of 102 patients with OHCA were included in this study. The study population were divided into two groups as survivor $(\mathrm{n}=43)$ and non-survivor $(\mathrm{n}=59)$ during follow-up. Complete blood cell counts, biochemical and blood gas analysis were recorded for all patients. Neutrophil to lymphocyte ratio (NLR) was calculated as the ratio of neutrophil to lymphocyte. CAR was calculated as the ratio of CRP to the albumin. Results: NLR $(\mathrm{P}=0.012)$, CAR $(\mathrm{P}<0.001)$ and serum lactate level $(\mathrm{P}=0.002)$ were significantly higher whereas lymphocyte $(\mathrm{P}=0.008)$ and serum albumin $(\mathrm{P}<0.001)$ were significantly lower in non-survivor group compared to survivor group. Multivariate logistic regression analysis showed that NLR (odds ratio [OR]: 1.044, 95\% confidence interval [CI]: 1.044-1.437, $\mathrm{P}=0.013$ ), CAR (OR: 1.971, 95\% CI: 1.327-2.930, $\mathrm{P}=0.001$ ), and lactate level (OR: 1.268, 95\% CI: 1.095-1.469, $\mathrm{P}=0.002$ ) were independent predictors of mortality. Conclusions: We have demonstrated for the first time that CAR was an independent predictor of in-hospital mortality in OHCA patients.
\end{abstract}

Prognostic Value of C-Reactive Protein to Albumin Ratio in Patients Resuscitated from Outof-Hospital Cardiac Arrest

\section{ABSTRACT}

Background: Despite major advances in basic and advanced life supports, patients who survived from out of hospital cardiac arrest (OHCA) has still poor prognosis. Several inflammatory parameters have been used to determine early and long-term prognosis in patients with OHCA. C-reactive protein-to-albumin ratio $(\mathrm{CAR})$ is also a novel marker of systemic inflammation. To our knowledge, there is no study evaluating the clinical importance of CAR in OHCA patients.

Aims: To evaluate the effect of CAR on mortality in patients with OHCA.

Methods: A total of 102 patients with OHCA were included in this study. The study population were divided into two groups as survivor $(\mathrm{n}=43)$ and non-survivor $(\mathrm{n}=59)$ during follow-up. Complete blood cell counts, biochemical and blood gas analysis were recorded for all patients. Neutrophil to lymphocyte ratio (NLR) was calculated as the ratio of neutrophil to lymphocyte. CAR was calculated as the ratio of CRP to the albumin.

Results: NLR $(\mathrm{P}=0.012)$, CAR $(\mathrm{P}<0.001)$ and serum lactate level $(\mathrm{P}=0.002)$ were significantly higher whereas lymphocyte $(\mathrm{P}=0.008)$ and serum albumin $(\mathrm{P}<0.001)$ were significantly lower in non-survivor group 
compared to survivor group. Multivariate logistic regression analysis showed that NLR (odds ratio [OR]: 1.044, 95\% confidence interval [CI]: 1.044-1.437, $\mathrm{P}=0.013$ ), CAR (OR: 1.971, 95\% CI: 1.327-2.930, $\mathrm{P}=0.001$ ), and lactate level (OR: 1.268, 95\% CI: 1.095-1.469, $\mathrm{P}=0.002$ ) were independent predictors of mortality.

Conclusions: We have demonstrated for the first time that CAR was an independent predictor of in-hospital mortality in OHCA patients.

Keywords: Out of hospital cardiac arrest, systemic inflammation, neutrophil to lymphocyte ratio, Creactive protein to albumin ratio, mortality.

\section{What's known?}

Cardiopulmonary resuscitation (CPR) improves the prognosis of patients with cardiac arrest (CA). Despite all technological improvements, the prognosis of patients with out of hospital cardiac arrest (OHCA) is still remains to be poor. Many inflammatory markers have been evaluated in these patients and demonstrated to be associated with poor prognosis.

\section{What's new?}

Recent studies have shown that C-reactive protein (CRP) to albumin ratio (CAR) is a novel and good marker of systemic inflammation. CAR reflects the balance between CRP and albumin which demonstrated to be more sensitive than using CRP and albumin separately. Although the importance of CAR has been evaluated in different patient population, to our knowledge, there is no study evaluating the importance of CAR in patients with OHCA. We have detected for the first time that CAR was an independent predictor of in-hospital mortality in OHCA patients.

\section{Review criteria}

\section{How did you gather, select and analyze the information you considered in your review?}

Patients who were brought to our emergency services due to OHCA and hospitalized in ICU after a successful CPR were investigated retrospectively. A total of 102 patients with OHCA were included in this study. The patient's data were obtained from the hospital database system retrospectively. CAR was calculated as the ratio of CRP to the albumin. The study population were divided into two groups as follows: survivor (n $=43)$ and non-survivor $(\mathrm{n}=59)$ during follow-up. The obtained characteristics and laboratory parameters were compared between two groups.

\section{Message for the clinic:}

\section{What is the 'take-home' message for the clinician?}

We have demonstrated that CAR was an independent predictor of in-hospital mortality in OHCA patients. Therefore, it may be useful when identifying the subjects under higher risk in patients with OHCA.

\section{1 | INTRODUCTION}

Cardiopulmonary resuscitation $(\mathrm{CPR})$ is the most important interventional procedure which contributes to the survival of patients with cardiac arrest (CA) and consists of basic and advanced life supports. ${ }^{1}$ Despite major advances in CPR and post-resuscitation care, patients surviving out of hospital cardiac arrest (OHCA) still carry poor prognosis including increased risk of mortality and morbidity. ${ }^{2,3}$ The whole-body ischemia during CA leads to systemic inflammatory response damage and reperfusion of this tissue triggers even more tissue injury which is defined as ischemia-reperfusion injury. ${ }^{4}$ Therefore, it is important to evaluate the prognosis after CPR in patients who survived from CA.

Systemic inflammation can be evaluated with many laboratory markers. One of these markers is neutrophil to lymphocyte ratio (NLR) which reflect the balance between neutrophils and lymphocytes. ${ }^{5}$ NLR play a crucial role in the ischemia-reperfusion injury. It has been demonstrated that higher NLR is associated with mortality in patients with OHCA. ${ }^{6}$ Nevertheless, C-reactive protein (CRP) to albumin ratio (CAR) is also a novel marker of systemic inflammation. It reflects the balance between CRP and albumin and detected to 
be more sensitive than CRP and albumin separately to evaluate the intense of inflammatory response. ${ }^{7}$ The clinical importance of CAR has been demonstrated in different patient population. ${ }^{8-10}$

Although previous studies investigated the association of NLR with prognosis in patients with OHCA, to our knowledge, there is no study evaluating the clinical importance of CAR in these patients. The aim of our study is to assess the effect of CAR on mortality in patients with OHCA.

\section{2 | METHODS}

Patients who were brought to our emergency services due to OHCA and hospitalized in ICU after a successful CPR were investigated retrospectively. Successful CPR was defined as acute respond to the CPR in the emergency department and transfer to the ICU for follow-up. Patients with trauma, systemic infection, intracranial hemorrhage, major surgery within the previous 14 days and malignancy were excluded from the study. Consequently, a total of 102 patients with OHCA were included in this study. Local ethic committee was approved the study design and the study was conducted in full accordance with the Declaration of Helsinki.

Blood samples were obtained routinely immediately after hospital admission. Complete blood cell (CBC) counts were measured with an automated blood cell counter. Neutrophil to lymphocyte ratio (NLR) was calculated as the ratio of neutrophil to lymphocyte. Platelet to lymphocyte ratio (PLR) was calculated as the ratio of platelet to lymphocyte. Also, arterial blood gas sampling and biochemical analyses including serum electrolytes, creatinine, aminotransferases, serum albumin and CRP was obtained from all patients. CAR was calculated as the ratio of CRP to the albumin.

Our study population were divided into two groups as follows: survivor $(\mathrm{n}=43)$ and non-survivor $(\mathrm{n}=$ 59) during follow-up. The archive records of all patients were investigated retrospectively, and baseline characteristics, blood gas samples and laboratory parameters were compared between two groups.

\section{1 | Statistical analysis}

Statistical analysis was conducted with SPSS 20 (SPSS, Chicago, IL). Kolmogorov-Smirnov test was used to determine the normality of distribution. Continuous variables were expressed as mean $\pm \mathrm{SD}$ or median (25-75 IQR) and compared with Student t test or Mann-Whitney U test according to the distribution. Categorical variables were expressed as number and percentages and compared with chi-square or Fisher's exact chi-square test. Multivariate logistic regression analysis with backward elimination was used to determine the independent predictors of mortality. Receiver operating characteristics (ROC) curve was performed to determine the area under curve (AUC) of variables for predicting mortality. The comparison of the AUCs were performed with Delong test. A $p$ value of less than 0.05 was considered as statistically significant.

\section{3 | RESULTS}

A total of 102 OHCA patients were included in this study. The mean age of the study population was were $61.0 \pm 12.5$ and $72.5 \%$ of the patients were male. Of these 102 patients, $59(57.8 \%)$ died during the hospital stay. The comparison of baseline characteristics of the survivor and non-survivor patients are listed in Table 1. It was found that the duration of ICU was significantly longer in survivor group compared to non-survivor group.

Comparison of the laboratory parameters of the study population is presented in Table 2. NLR (4.5 [3.3-5.9] vs. $2.9[1.0-5.7], \mathrm{P}=0.012), \mathrm{CRP}(6.4[1.9-11.8]$ vs. $1.9[0.8-4.5], \mathrm{P}<0.001), \mathrm{CAR}(1.6[0.5-3.3]$ vs. 0.5 [0.2-1.3], $\mathrm{P}<0.001)$ and lactate level (7 [3.8-11.2] vs. $4.4[2.5-7.0], \mathrm{P}=0.002)$ were significantly higher whereas lymphocyte (2.6 [1.5-3.2] vs. 3.5 [1.9-5.8], $\mathrm{P}=0.008)$ and serum albumin level $(3.4 \pm 0.6$ vs. $3.8 \pm$ $0.4, \mathrm{P}<0.001)$ were significantly lower in non-survivor group.

The area under the curves (AUC) of CAR, CRP and albumin were compared with each other to determine whether there was an additional benefit of using CAR on CRP and albumin for predicting in-hospital mortality. The AUC of CAR was significantly higher than both the AUC of CRP (0.738 vs 0.717, $\mathrm{P}=$ 
$002)$ and albumin $(0.738$ vs. $0.611, \mathrm{P}<0.001)$ for predicting in-hospital mortality (Figure 1). CAR [?] 4.6 predicted in-hospital mortality with a sensitivity of $64.4 \%$ and specificity of $76.7 \%$.

Multivariate logistic regression analysis was performed to determine the independent predictors of mortality. NLR (odds ratio [OR]: 1.044, 95\% confidence interval [CI]: 1.044-1.437, $\mathrm{P}=0.013$ ), CAR (OR: 1.971, 95\%

CI: $1.327-2.930, \mathrm{P}=0.001)$, and lactate level (OR: $1.268,95 \% \mathrm{CI}: 1.095-1.469, \mathrm{P}=0.002$ ) were found to be the independent predictors of mortality (Table 3 ).

\section{4 | DISCUSSION}

In this study, we investigated the prognostic value of CAR in patients with OHCA. The main finding of our study was that CAR was significantly higher in non-survivor group compared to in survivor group. Also, CAR was an independent predictor of in-hospital mortality. To our knowledge, this is the first study demonstrating the independent relationship between CAR and in hospital mortality in patients with OHCA.

The extensive ischemia during CA leads to the activation of systemic inflammation. ${ }^{4}$ After that, some complex pathophysiological changes occur such as free oxygen radical production, increased vascular permeability and disruption of the blood-brain barrier. All these changes cause systemic ischemia and multi organ failure. ${ }^{4,11}$ Therefore, it is very important to assess the prognosis after CPR and to predict survival early and accurately in patients who survived from CA.

After cardiac arrest, lactate accumulation occurs due to interruption of blood flow to metabolically active tissues and decreased tissue oxygenation. ${ }^{12}$ Therefore, serum lactate level, an easily obtainable marker from blood gas analysis, was used in several studies for predicting poor outcomes in patients with OHCA. ${ }^{12-15}$ It was found that higher lactate level on admission was associated with unfavorable outcomes and poor prognosis. Similar to these studies, we also found that serum lactate level on admission was significantly higher in non-survivor group compared to survivor group. In addition, we detected that serum lactate level was an independent predictor of mortality. These results suggest that on admission serum lactate level may be used as a beneficial tool for predicting in-hospital mortality in patients with OHCA.

Because a widely inflammatory process is triggered in patients with cardiac arrest, previous studies have frequently investigated the importance of inflammatory markers. ${ }^{16-20}$ NLR, a cheap and an easily detectable marker from complete blood cell, is a combination of two independent markers of systemic inflammation. ${ }^{5}$ It was demonstrated that NLR is a stronger parameter that other white blood cell subtypes for evaluating the inflammatory process. ${ }^{21}$ Therefore, it is one of the most used inflammatory markers in daily practice. Weiser et al. ${ }^{6}$ found that NLR at admission predicted mortality independently in patients with OHCA. Similar to this study, we also found that NLR at hospital admission was an independent predictor of mortality in these patients. These results suggest that in hospital prognosis may be poorer in patients with OHCA where a more intense inflammatory process is triggered.

CRP and albumin are biochemical markers of systemic inflammation. The clinical usefulness of them have been investigated in previous studies. ${ }^{22,23}$ Increased level of CRP and decreased level of albumin were found to be associated with poor outcomes in patients with CA. CAR is a novel marker of systemic inflammation and can be obtained easily from biochemical analysis. ${ }^{7}$ It reflects the balance between CRP and albumin and has been found that the combination of these two markers are stronger and more consistent than CRP or albumin alone for predicting outcomes. ${ }^{10}$ Although previous studies separately investigated the CRP and albumin levels in patients with CA. ${ }^{22,23}$ To our knowledge, there is no study evaluating CAR in patients with OHCA. In the present study, we investigated the CAR level in addition to the NLR in patients with OHCA. We found that CAR was significantly higher in non-survivor group compared to survivor group. Also, CAR was an independent predictor of in-hospital mortality. Moreover, we found that the AUC of CAR was significantly higher than both the AUCs of CRP and albumin for predicting in-hospital mortality. These results suggest that CAR has a better prognostic role than either CRP or albumin alone for predicting poor outcomes in patients with OHCA. In addition, it can be concluded that CAR is a good clinical marker for predicting in-hospital mortality after surviving from OHCA. 


\section{1 | Limitations}

Our study had some limitations. The most important limitation of our study was small sample size and its retrospective design. Second, other inflammatory markers such as cytokines were not investigated in this study. Third, long term outcomes were not evaluated. It could be interesting to determine the effect of CAR on long term outcomes. Further prospective studies with larger participant is needed to better elucidate the prognostic role of CAR in patients with OHCA.

\section{5 | CONCLUSION}

CAR is a novel marker of systemic inflammation. In this study, we have demonstrated for the first time that CAR was an independent predictor of in-hospital mortality in OHCA patients.

\section{ACKNOWLEDGEMENTS}

No acknowledgements

\section{CONFLICT OF INTEREST}

The authors declare that they have no competing interests.

\section{FUNDING}

The authors received no financial support for the research, authorship, and/or publication of this article.

\section{AUTHOR CONTRIBUTIONS}

Concept - T.B.T., G.P., F.T.B., B.C.K., M.T.; Design - T.B.T., G.P., F.T.B., B.C.K., M.T.; Supervision - T.B.T., G.P., M.T.; Resources - T.B.T., G.P., F.T.B., B.C.K., M.T.; Data Collection and/or Processing - T.B.T., G.P., F.T.B., B.C.K., M.T.; Analysis and/or Interpretation - T.B.T., F.T.B., B.C.K.; Literature Search - T.B.T., G.P., F.T.B., B.C.K., M.T.; Writing Manuscript - T.B.T., G.P., B.C.K.; Critical Review - T T.B.T., G.P., F.T.B., B.C.K., M.T.

\section{REFERENCES}

1. Link MS, Berkow LC, Kudenchuk PJ, et al. Part 7: Adult Advanced Cardiovascular Life Support: 2015 American Heart Association Guidelines update for cardiopulmonary resuscitation and emergency cardiovascular care. Circulation. 2015;132(18 Suppl 2):S444-S464.

2. Du L, Zheng K, Feng L, et al. The First national survey on Practices of Neurological Prognostication after Cardiac Arrest in China, still a lot to do. Int J Clin Pract. 2020 Oct 23:e13759. doi: 10.1111/ijcp.13759. Epub ahead of print..

3. Nolan JP, Soar J, Cariou A, et al. European resuscitation council and European Society of Intensive Care Medicine Guidelines for post-resuscitation care 2015: section 5 of the European Resuscitation Council Guidelines for resuscitation. Resuscitation 2015;95:202-222.

4. Widgerow AD. Ischemia-reperfusion injury. Ann Plast Surg 2014;72:253-260.

5. Zheng J, Cai J, Li H, et al. Neutrophil to Lymphocyte Ratio and Platelet to Lymphocyte Ratio as Prognostic Predictors for Hepatocellular Carcinoma Patients 239 with Various Treatments: a MetaAnalysis and systematic Review. Cell Physiol Biochem 2017;44:967-981.

6. Weiser C, Schwameis M, Sterz F, et al. Mortality in patients resuscitated from out-of-hospital cardiac arrest based on automated blood cell count and neutrophil lymphocyte ratio at admission. Resuscitation 2017;116:49-55

7. Park JE, Chung KS, Song JH, et al. The C-Reactive Protein/Albumin Ratio as a Predictor of Mortality in Critically Ill Patients. J Clin Med 2018;7:333.

8. Cui X, Jia Z, Chen D, et al. The prognostic value of the C-reactive protein to albumin ratio in cancer: An updated meta-analysis. Medicine (Baltimore). 2020;99(14):e19165.

9. Oh TK, Ji E, Na HS, et al. C-Reactive Protein to Albumin Ratio Predicts 30-Day and 1-Year Mortality in Postoperative Patients after Admission to the Intensive Care Unit. J Clin Med. 2018;7:39. 
10. Tanriverdi Z, Gungoren F, Tascanov MB, et al. Comparing the Diagnostic Value of the C-Reactive Protein to Albumin Ratio with Other Inflammatory Markers in Patients With Stable Angina Pectoris. Angiology 2020;71:360-365.

11. Amnuaypattanapon K, Thanachartwet V, Desakorn V, et al. Predictive model of return of spontaneous circulation among patients with out-of-hospital cardiac arrest in Thailand: The WATCH-CPR Score. Int J Clin Pract. 2020 Jul;74(7):e13502.

12. Momiyama Y, Yamada W, Miyata K, et al. Prognostic values of blood $\mathrm{pH}$ and lactate levels in patients resuscitated from out-of-hospital cardiac arrest. Acute Med Surg 2017;4:25-30.

13. Kliegel A, Losert H, Sterz F, et al. Serial lactate determinations for prediction of outcome after cardiac arrest. Medicine (Baltimore) 2004; 83: 274-279.

14. Adrie C, Cariou A, Mourvillier B, et al. Predicting survival with good neurological recovery at hospital admission after successful resuscitation of out-ot-hospital cardiac arrest: the OHCA score. Eur Heart J 2006;27:2840-2845.

15. Cocchi MN, Salciccioli J, Yankama T, et al. Predicting Outcome After Out-of-Hospital Cardiac Arrest: Lactate, Need for Vasopressors, and Cytochrome c. J Intensive Care Med. 2019;29:885066619873315.

16. Patel VH, Vendittelli P, Garg R, et al. Neutrophil-lymphocyte ratio: A prognostic tool in patients with in-hospital cardiac arrest. World J Crit Care Med. 2019;8:9-17.

17. Kim HJ, Park KN, Kim SH, et al. Association between the neutrophil-to-lymphocyte ratio and neurological outcomes in patients undergoing targeted temperature management after cardiac arrest. J Crit Care 2018;47:227-231.

18. Başer K, Baş HD, Attaluri $\mathrm{P}$, et al. Changes in neutrophil-to-lymphocyte ratios in postcardiac arrest patients treated with targeted temperature management. Anatol J Cardiol 2017;18:215-222.

19. Cakmak S, Sogut O, Albayrak L, et al. Serum Copeptin Levels Predict the Return of Spontaneous Circulation and the Short-Term Prognosis of Patients with Out-of-Hospital Cardiac Arrest: A Randomized Control Study. Prehosp Disaster Med 2020;35:120-127.

20. Jou C, Shah R, Figueroa A, et al. The Role of Inflammatory Cytokines in Cardiac Arrest. J Intensive Care Med 2020;35:219-224.

21. Azab B, Zaher M, Weiserbs KF, et al. Usefulness of neutrophil to lymphocyte ratio in predicting shortand long-term mortality after non-ST-elevation myocardial infarction. Am J Cardiol. 2010;106:470476.

22. Dell'anna AM, Bini Viotti J, Beumier M, et al. C-reactive protein levels after cardiac arrest in patients treated with therapeutic hypothermia. Resuscitation 2014;85:932-938.

23. Matsuyama T, Iwami T, Yamada T, et al. Effect of Serum Albumin Concentration on Neurological Outcome After Out-of-Hospital Cardiac Arrest (from the CRITICAL [Comprehensive Registry of Intensive Cares for OHCA Survival] Study in Osaka, Japan). Am J Cardiol 2018;121:156-161.

Table 1. Comparison of the baseline characteristics of the study population

\begin{tabular}{llll}
\hline & $\begin{array}{l}\text { Survivor group }(\mathbf{n}= \\
\mathbf{4 3})\end{array}$ & $\begin{array}{l}\text { Non-survivor group }(\mathbf{n} \\
\mathbf{5 9 9}\end{array}$ & $\mathbf{P}$ \\
\hline Age, years & $60.3 \pm 11.9$ & $61.5 \pm 13.0$ & 0.616 \\
Male gender (\%) & $29(67.4)$ & $45(76.3)$ & 0.324 \\
HT (\%) & $6(14)$ & $9(15.3)$ & 0.855 \\
DM (\%) & $6(14)$ & $8(13.6)$ & 0.954 \\
CAD (\%) & $14(32.6)$ & $20(33.9)$ & 0.887 \\
COPD (\%) & $2(4.7)$ & $2(3.4)$ & 0.746 \\
Duration of CPR, min. & $30(20-35)$ & $25(15-30)$ & 0.510 \\
Duration of ICU, days & $6(3-24)$ & $2(1-6)$ & $<\mathbf{0 . 0 0 1}$ \\
Duration of MV, days & $1(1-13)$ & $2(1-6)$ & 0.861 \\
\hline
\end{tabular}

Note: Data are presented as n $(\%)$, mean $\pm \mathrm{SD}$ and the median $\left(25^{\text {th }}-75^{\text {th }}\right.$ interquartile range). The P-value 
was calculated using the chi square (categorical variables), student t test or Mann-Whitney U- (numerical variables) tests.

Abbreviations: M, male; F, female; HT, hypertension; DM, diabetes mellitus; CAD, coronary artery disease; COPD, chronic obstructive pulmonary disease; CPR, cardiopulmonary resuscitation; ICU, intensive care unit; MV, mechanical ventilator

Table 2. Comparison of the laboratory parameters of the study population

\begin{tabular}{llll}
\hline & $\begin{array}{l}\text { Survivor group }(\mathbf{n}= \\
\mathbf{4 3})\end{array}$ & $\begin{array}{l}\text { Non-survivor group } \mathbf{n} \\
\mathbf{5 9 9}\end{array}$ & $\mathbf{P}$ \\
\hline WBC $\left(\mathrm{x} 10^{3} / \mathrm{mL}\right)$ & $14.9 \pm 6.1$ & $15.2 \pm 7.9$ & 0.850 \\
Neutrophil $\left(\mathrm{x} 10^{3} / \mathrm{mL}\right)$ & $9.5(4.3-14.0)$ & $9.6(6.9-16.5)$ & 0.169 \\
Lymphocyte & $3.5(1.9-5.8)$ & $2.6(1.5-3.2)$ & $\mathbf{0 . 0 0 8}$ \\
$\left(\mathrm{x} 10^{3} / \mathrm{mL}\right)$ & & & \\
Monocyte $\left(\mathrm{x} 10^{3} / \mathrm{mL}\right)$ & $0.6(0.4-0.9)$ & $0.5(0.3-0.8)$ & 0.261 \\
Hemoglobin $(\mathrm{g} / \mathrm{dL})$ & $13.6 \pm 2.5$ & $13.3 \pm 2.5$ & 0.551 \\
Platelet $\left(\mathrm{x} 10^{3} / \mathrm{mL}\right)$ & $263(210-313)$ & $243(180-317)$ & 0.169 \\
MCV $(\mathrm{fL})$ & $91.4 \pm 9.1$ & $90.0 \pm 6.8$ & 0.364 \\
RDW $(\%)$ & $12.8 \pm 1.5$ & $14.9 \pm 10.5$ & 0.195 \\
NLR & $2.9(1.0-5.7)$ & $4.5(3.3-5.9)$ & $\mathbf{0 . 0 1 2}$ \\
PLR & $79(37-170)$ & $92(61-176)$ & 0.146 \\
Glucose, $\mathrm{mg} / \mathrm{dl}$ & $250(160-318)$ & $304(175-407)$ & 0.272 \\
Creatinine, mg/dl & $1.2 \pm 0.4$ & $1.4 \pm 0.4$ & 0.144 \\
Na $(\mathrm{mEq} / \mathrm{L})$ & $138.6 \pm 5.4$ & $139.5 \pm 4.7$ & 0.372 \\
K $(\mathrm{mEq} / \mathrm{L})$ & $4.4 \pm 0.6$ & $4.7 \pm 0.9$ & 0.107 \\
Ca $(\mathrm{mEq} / \mathrm{L})$ & $10.4 \pm 11.0$ & $8.5 \pm 0.8$ & 0.197 \\
AST, IU & $68(33-163)$ & $102(42-370)$ & 0.067 \\
ALT, IU & $63(31-127)$ & $72(33-267)$ & 0.102 \\
CRP, & $1.9(0.8-4.5)$ & $6.4(1.9-11.8)$ & $<\mathbf{0 . 0 0 1}$ \\
Albumin, g/dl & $3.8 \pm 0.4$ & $3.4 \pm 0.6$ & $<\mathbf{0 . 0 0 1}$ \\
CAR & $0.5(0.2-1.3)$ & $1.6(0.5-3.3)$ & $<\mathbf{0 . 0 0 1}$ \\
PH & $7.2 \pm 0.2$ & $7.1 \pm 0.2$ & 0.056 \\
Lactate & $4.4(2.5-7.0)$ & $7(3.8-11.2)$ & $\mathbf{0 . 0 0 2}$ \\
\hline
\end{tabular}

Abbreviations: WBC, white blood cell; MCV, mean corpuscular volume; RDW, red cell distribution width; NLR, neutrophil to lymphocyte ratio; PLR, platelet to lymphocyte ratio; Ns, sodium; K, potassium; AST, aspartate aminotransferase; ALT, alanine aminotransferase; CRP, C-reactive protein; CAR, CRP to albumin ratio.

Table 3. Independent predictors of the mortality

\begin{tabular}{llll}
\hline & OR & $\mathbf{9 5 \%}$ CI & P \\
\hline NLR & 1.044 & $1.044-1.437$ & 0.013 \\
CAR & 1.971 & $1.327-2.930$ & 0.001 \\
Lactate & 1.268 & $1.095-1.469$ & 0.002 \\
\hline
\end{tabular}

Abbreviations: OR, odds ratio; CI, confidence interval; CAR, CRP to albumin ratio; NLR, neutrophil to lymphocyte ratio

Entered variables: age, gender, hypertension, diabetes mellitus, coronary artery disease, duration of car- 
diopulmonary resuscitation, hemoglobin, platelet, creatinine, glucose, $\mathrm{pH}$, lactate, CAR, NLR

\section{Figure Legends}

Figure 1. ROC curve of C-reactive protein, albumin and C-reactive protein to albumin ratio for predicting in-hospital mortality

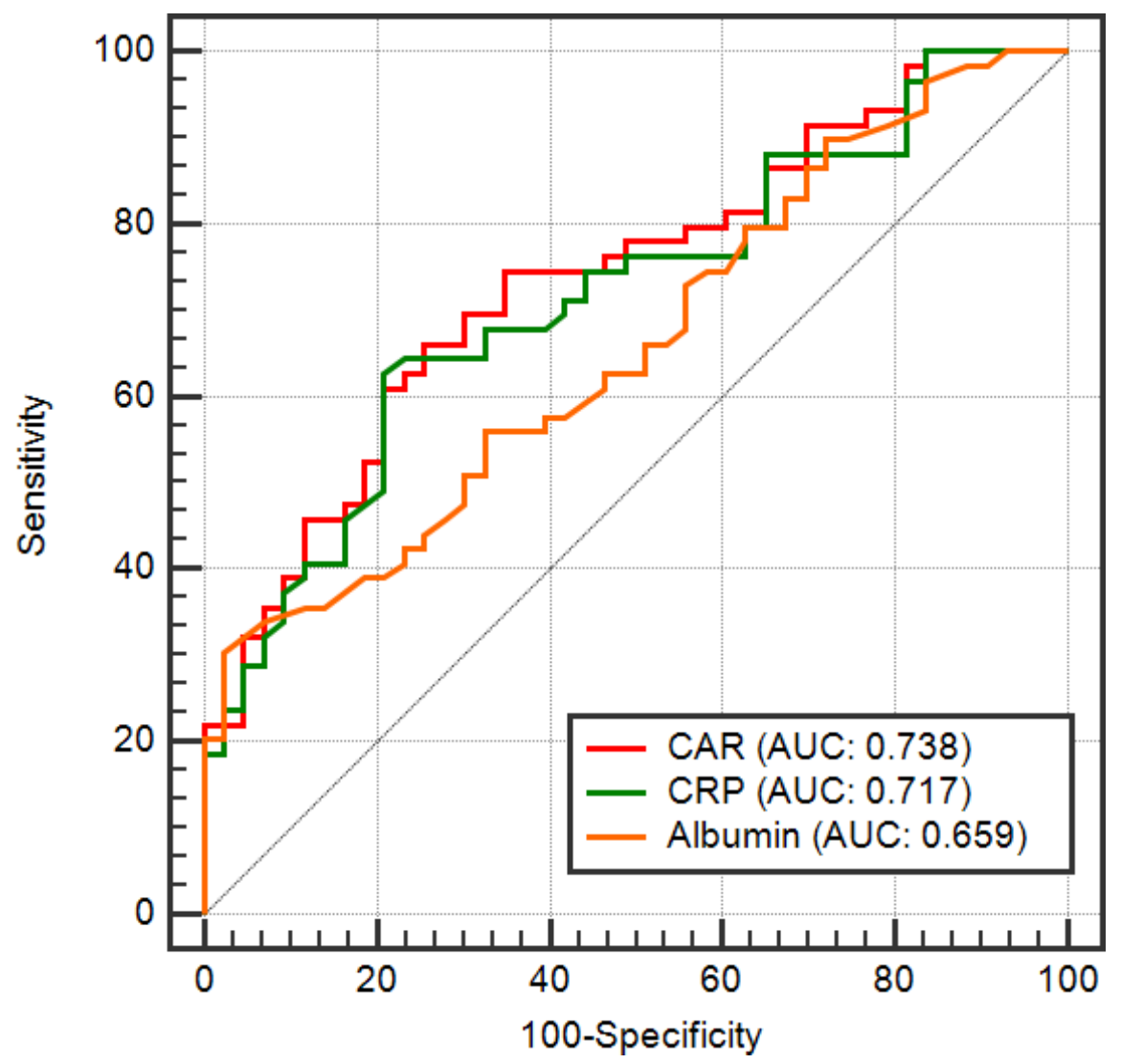

